Please cite as: Unkelbach, C., \& Greifeneder, R. (2018). Experiential fluency and declarative advice jointly inform judgments of truth. Journal of Experimental Social Psychology, 79, 78-86.

https://doi.org/10.1016/j.jesp.2018.06.010

Experiential fluency and declarative advice jointly inform judgments of truth

Christian Unkelbach

Universität zu Köln

Rainer Greifeneder

Universität Basel

Word Count: 6957

(excluding references and abstract)

Corresponding Author:

Christian Unkelbach

Social Cognition Center Cologne

Universität zu Köln

Richard-Strauss-Str. 2

50931 Köln

Germany

Email: christian.unkelbach@uni-köln.de 
Fluency_and_Advice 2

\begin{abstract}
Processing fluency, the experienced ease of ongoing mental operations, influences judgments such as frequency, monetary value, or truth. Most experiments keep to-be-judged stimuli ambiguous with regards to these judgment dimensions. In real life, however, people usually have declarative information about these stimuli beyond the experiential processing information. Here, we address how experiential fluency information may inform truth judgments in the presence of declarative advice information. Four experiments show that fluency influences judged truth even when advice about the statements' truth is continuously available and labeled as highly valid; the influence follows a linear cue integration pattern for two orthogonal cues (i.e., experiential and declarative information). These data underline the importance of processing fluency as an explanatory construct in real-life judgements and support a cue integration framework to understand fluency effects in judgment and decision making.
\end{abstract}




\section{Experiential fluency and declarative advice jointly inform judgments of truth}

Processing fluency is the experiential component of mental operations such as perceiving, storing, retrieving, or generating information (see Unkelbach \& Greifeneder, 2013). This fluency experience influences judgments and evaluations from basic dimensions such as stimuli's frequency (e.g., Tversky \& Kahneman, 1973), likeability (e.g., Reber, Winkielman, \& Schwarz, 1998), or size (e.g., Reber, Zimmermann, \& Wurtz, 2004), to more complex judgments such as the truth of statements (e.g., Hansen, Dêchene, \& Wänke, 2008), the ability of persons (e.g., Greifeneder et al., 2010), or companies' economic value (e.g., Hertwig, Herzog, Schooler \& Reiner, 2008). Processing fluency increases judgments and evaluations on the relevant dependent variable. High fluency stimuli are judged to be more frequent, more likeable, and bigger; and if the relevant information is processed more fluently, statements appear more true, target persons more capable, and companies more valuable (and vice versa for low fluency; however, for reversals of this relation, see Olds \& Westerman, 2012; Unkelbach, 2006, 2007).

A surprisingly neglected question in these research lines is how processing fluency influences judgments when other information is available; for example, knowledge about a person's ability or a company's value. That is, how is experiential fluency information integrated with available declarative information? Many studies assume, implicitly or explicitly, that people rely either on fluency or on other available information. This either-or perspective is particularly apparent in studies investigating moderators of fluency use, which often investigate one condition in which fluency information, and another condition in which semantic content information, is expected to be relied on in judgment (see Greifeneder, Bless, \& Pham, 2011). The either-or perspective is also prominently present in studies on fluency and judged truth: Researchers typically choose statements for which participants have little or no knowledge. As Dechêne, Stahl, Hansen, and Wänke (2010) stated in their review: "Statements have to be ambiguous, that is, participants have to be uncertain about their truth status because otherwise the statements' truthfulness will be judged on the basis of their knowledge" (p. 239). Two examples of studies that investigated additional informational cues for judging truth illustrate this distinction. Fazio, Brashier, Payne, and Marsh (2015) had participants 
judge statements for which they should have knowledge, and Unkelbach and Stahl (2009) had information presented by a truthful and untruthful source. Both studies employed multinomial processing trees which necessitate this either-or distinction (see Fazio et al., 2015; Fig. 2 and 3; Unkelbach \& Stahl, 2009; Fig. 1) and also translate the distinction into their discussion "): "The results of two experiments suggest that people sometimes fail to bring their knowledge to bear and instead rely on fluency as a proximal cue." (Fazio et al., 2015, p. 999).

In contrast, we assume that processing fluency influences judgments and evaluations jointly with other available information. This prediction is central to the fluency model by Unkelbach and Greifeneder (2013). They argued that fluency effects are best described using the lens model by Brunswik (1952, 1955; see Kareleia \& Hogarth, 2008, for an overview). The lens model perspective holds that any given judgment is influenced by informational cues which are weighted according to their subjective validity and then linearly integrated. Thus, both processing fluency and other information (e.g., knowledge) should jointly influence judgments and evaluations. ${ }^{1}$

\section{Experiential fluency effects in the presence of declarative information}

Most experiments carefully control their materials as such that the sole systematic difference between stimuli is indeed processing fluency. For example, participants judge statements such as "Osorno is in Chile" as more likely true when presented in high fluency colors (i.e., blue and red) compared to low fluency colors (i.e., green and yellow; Reber \& Schwarz, 1999; Unkelbach, 2007). Random assignment of statements to the high/low fluency conditions ensures that changes on the relevant DV are directly interpretable as fluency effects.

This typical setup does not allow gauging the contribution of processing fluency when other information is available; for example, when participants factually know that Osorno is a city in Chile (it is). Intuitively, the statement's font color should have no impact when one has relevant knowledge about the task. However, this intuition restricts fluency influences to cases when there is no other information available about stimuli in terms of their ability, monetary value, or truth.

The deeply rooted intuition that fluency should play no role if declarative information is present derives from a Cartesian dualism, which gives knowledge and reasoning a priori precedence 
over experiential information; that is, given knowledge about a matter, a "rational" judge should not be influenced by experienced processing fluency. However, if one drops the a priori Cartesian ordering, it becomes less clear why experiential (e.g., fluency) and declarative (e.g., semantic knowledge) information should not have joint influences on judgments. In a lens model view (Brunswik, 1952, 1955), any cue may "rationally" influence judgments, as long as it has an ecological correlation with the to-be-judged criterion; and fluency might be such an ecologically valid cue (see Greifeneder, Bless, \& Scholl, 2013; Hertwig et al., 2008; Reber \& Unkelbach, 2010). Thus, even if someone believes to know that Osorno is in Chile, presenting this piece of trivia in a difficult-toprocess way may lead to lower truth ratings just because the statement feels false.

Fazio and colleagues (2015) showed this point when participants evaluated the truth of repeated (i.e., high fluency) and new (i.e., low fluency) statements. For half of the statements, participants might have known the factual truth (60\% correct answers in a norming sample; p. 994), while the other half were more difficult (only $5 \%$ correct answers in a norming sample). Fazio and colleagues observed a reliable fluency effect for both kinds of statements. However, the either-or perspective and the Cartesian precedence of "knowing" over "feeling" is still present in Fazio and colleagues' argument (see above). It follows from their conclusions ( $p .999$ ) that given people bring knowledge to bear, there should be no fluency effect. Here we go a critical step further and show that even if other information is clearly available for the judgment, people are still influenced by processing fluency.

\section{The present research}

Building on the model by Unkelbach and Greifeneder (2013), we argue that experiential fluency information and declarative information jointly influence judgments. In the following four experiments, we manipulated an experiential cue and a declarative cue. First, participants either judged new statements or repeated statements. As repetition influences processing ease (Feustel, Shiffrin, \& Salasoo, 1983), this creates statements of relative low and high fluency, respectively. Second, participants received advice whether the to-be-judged statement is "true" or "false." In addition, the provided advice was labelled to be 50\%, 60\%, or 70\% correct (Exp. 1 and 2), 70\%, 80\%, 
or $90 \%$ correct (Exp. 3), and $50 \%$ or $100 \%$ correct (Exp. 4). Thus, we did not rely on participants' knowledge about the statements. While appealing, participants' private knowledge is difficult to control experimentally; that is, as suggested by Fazio and colleagues (2015), even if participants have knowledge, they might fail to retrieve it. Here, the declarative advice information was consistently present. We expected processing fluency to influence truth judgments at all levels of the additional cue information.

For all experiments, we report all data exclusions, all manipulations, and all measures. We only report test statistics that are significant; we report non-significant results only when they are theoretically relevant. We report partial eta-square as effect size indicator and its $95 \%$ confidence intervals, based on the formulas in the SAS macro for effect size estimation by Kromrey and Bell (2010). Sample sizes are based on the authors' previous experiments with repetition-induced truth effects and data collection was always completed before any analyses. Raw data and analysis scripts are available online (https://osf.io/v2gmb/files/).

\section{Experiment 1}

Experiment 1 aimed to show the joint influence of processing fluency and declarative information on truth judgments. While reading new or repeated statements, participants got information from advisors about the statements' truth or falsity with a stated $50 \%, 60 \%$, or $70 \%$ validity. The main dependent variable was the mean probability of a "true" judgment (PTJ; see Unkelbach \& Rom, 2017). As manipulation check for processing fluency, we measured response latencies with the assumption that faster responses should map onto more fluent processing (e.g., Scholl, Greifeneder, \& Bless, 2014).

\section{Method}

Materials. We used 120 difficult trivia statements that were successfully employed in previous research (e.g., Hilbig, 2012; Unkelbach \& Stahl, 2009). Half of the statements were factually true and half were factually false. Knowledge influences on truth judgments are usually small (see 
Hilbig, 2012). A VisualBasic program controlled random assignments, instructions, stimulus presentations, and dependent variable assessments.

Participants and Design. Twenty-nine students (average age: 24.28 years; 19 female, 10 male) participated for payment or course credit. Within participants, we orthogonally manipulated repetition (repeated vs. new), advice ("true" vs. "false"), and advice validity (50\% vs. $60 \%$ vs. $70 \%$ "correct"). Statements were randomly assigned anew for each participant to these conditions (i.e., 10 statements per condition). Thus, the design has 3480 observations; this yields power to detect effects of Cohen's $d>.375$ with a Type I error probability of .05, and a Type II error probability of .20, for all relevant main effects and interactions (Westfall, Kenny, \& Judd, 2014; computed with PANGEA: Westfall, 2018). However, factual power is substantially higher because of the reliability gains due to the repeated measurements (Unkelbach \& Topolinski, 2017; Vanhove, 2017).

Procedure. The procedure followed a typical truth judgment study (see Dechêne et al., 2010). Upon arriving, participants read and signed an informed consent sheet. If they agreed to participate, experimenters seated them in front of a computer and started the VisualBasic program. The program informed participants that they would take part in a study investigating influences on judging the truth of statements. They would first see a presentation of statements; then they would do a distractor task. Next, they would be asked to judge a large set of statements as "true" or "false".

The presentation phase showed 60 randomly selected statements (for each participant anew) with restrictions implemented according to the design. A statement was on screen for $3.5 \mathrm{~s}$. The next statement followed after a delay of $1 \mathrm{~s}$. Next, participants started a filler task, which lasted between 6 to 12 minutes.

Then the judgment phase followed. The program informed participants that they would have to judge statements as true or false. In addition, they would see the advice of another person whether the statement is true or false. There would be three advisors labeled GALI, MEDI, and HOPI with correctness levels $50 \%, 60 \%$, and $70 \%$, respectively. How they used this advice would be up to themselves. After reading the instructions, participants started the judgments. To be sure, the advice level was clearly explained as such that $50 \%$ means "Out of 10 statements, Gali judges 5 correctly and 
5 incorrectly. The advisor is guessing." The same was stated for 6 out of 10 for Medi and 7 out of ten for Hopi, without the guessing part. Thus, although we use the term advice validity in the following, $50 \%$ correctness in a binary "true"/"false" advice translates to zero validity in a measurement sense. Participants used two marked keys on the keyboard for their "true"/"false" decisions. The program presented the statements in the upper third of the screen and together with the advisor centered below the statement. We used a schematic face for all three advisors together with a speech bubble stating: "GALI/MEDI/HOPI says: This statement is true/false". and the $50 \% / 60 \% / 70 \%$ validity of the advice present below the true/false information within the speech bubble, with the names, direction, and validity varying according to the assigned status of a statement. Together, statement, face, and speech bubble filled the middle $33 \%$ of the screen. The program presented 120 statements according to the design (i.e., 60 statements repeated from the presentation phase, and 60 new statements) and recorded decisions and latencies. Upon completion, the experimenter thanked and debriefed participants.

\section{Results}

We excluded data from two participants who indicated German as their third language, leaving 27 participants in the analysis. This exclusion did not affect any statistical conclusions. We calculated participants' mean PTJs for the twelve within-conditions.

PTJs. Figure 1 presents these mean PTJs and we analyzed these data with a repetition (repeated vs. new), advice ("true" vs. "false"), and advice validity (50\% vs. $60 \%$ vs. $70 \%$ "correct") repeated-measures ANOVA, with a profile analysis comparing the successive validity levels. Higher values imply higher probabilities to respond "true".

First, as Figure 1 shows, we replicated a standard truth effect: participants had higher PTJs for repeated $(M=0.655, S D=0.248)$ compared to new statements $(M=0.487, S D=0.260), F(1$, 26) $=18.47, p<.001, \eta_{\mathrm{p}}^{2}=.415,95 \% \mathrm{Cl}[.165, .577]$. Second, participants followed the advice: participants had higher PTJs for statements that advisors stated to be "true" $(M=0.670, S D=0.231)$ compared to "false" $(M=0.472, S D=0.266), F(1,26)=26.53, p<.001, \eta^{2}{ }_{p}=.505,95 \% \mathrm{Cl}[.255, .645]$. This advice main effect was qualified by an interaction with advice validity: higher validity led to 
higher/lower participants' PTJs, depending on the "true"/"false" status of the advice cue, $F(2$, $52)=13.39, p<.001, \eta_{p}^{2}=.340,95 \% \mathrm{Cl}[.155, .465]$. This interaction effect was mainly due to PTJ increases (decreases) for "true" advice ("false" advice) from the $50 \%$ to the $60 \%$ level, $F(1,26)=13.28$, $p=.001, \eta_{p}^{2}=.338,95 \% \mathrm{Cl}[.100, .515]$, while the interaction was less pronounced for PTJ increases (decreases) from the $60 \%$ to the $70 \%$ level and no longer significant, $F(1,26)=2.49, p=.127, \eta^{2}{ }_{p}=.087$, $95 \% \mathrm{Cl}[.000, .274]$

We also analyzed PTJs separately at $50 \%$ and $70 \%$ advice validity; at $50 \%$ validity, advice did not influence participants' PTJs, $F(1,26)=1.32, p=.260$, but repetition did, $F(1,26)=19.33, p<.001$, $\eta_{\mathrm{p}}^{2}=.426,95 \% \mathrm{Cl}[.175, .585]$. At $70 \%$, the repetition influence was reduced but still present, $F(1$, $26)=8.63, p=.007, \eta_{p}^{2}=.249,95 \% \mathrm{Cl}[.044, .440]$, while advice had a stronger impact, $F(1,26)=28.91$, $p<.001, \eta^{2}{ }_{p}=.527,95 \% \mathrm{Cl}[.279, .661]$. Yet, although the difference between new and repeated statements was lower in the $70 \%$ validity cases compared to the $50 \%$ validity cases $\left(M_{\text {diff }}=.133\right.$, $S D=.385)$, this reduction of the repetition effect was not different from zero, $t(26)=1.80, p=.084$.

Latencies. We set values larger than 7500 to 7500 and values lower than 1000 to 1000 . The same ANOVA as for the PTJs showed two main effects: Participants responded faster to repeated $(M=3911, S D=1329)$ compared to new statements $(M=4426, S D=1211), F(1,26)=21.88, p<.001$, $\eta_{p}^{2}=.457,95 \% \mathrm{Cl}[.204, .609] ;$ and participants responded faster given advice that statements are "true" ( $M=4082, S D=1319)$ rather than "false" $(M=4254, S D=1270), F(1,26)=9.72, p=.004, \eta^{2}{ }_{p}=.272$, $95 \% \mathrm{Cl}[.057, .460]$.

\section{Discussion}

Experiment 1 showed several points. First, processing fluency manipulated via repetition influenced truth judgments even when explicit advice was continuously present; and as the substantial influence of advice shows, this information was also correctly encoded. Supporting a lens model view, people seemingly used an experiential fluency cue jointly with a declarative advice cue.

Second, fluency influenced judgments on all levels of advice. Fluency had more impact when advice was at chance level $\left(\eta^{2}{ }_{p}=.426\right)$ compared to highly valid advice $\left(\eta^{2}=.249\right)$. Statistically, 
however, this decrease was not significant; although the present power level may not be sufficient to detect this interaction.

Third, the response latencies as a fluency manipulation check showed a clear effect of repetition. In addition, positive advice ("true") led to faster responses than negative advice ("false"); while this pattern follows from models of evaluative classification that predict faster processing of positive information (e.g., Unkelbach et al., 2008; Unkelbach et al., 2010; Topolinski \& Deutsch, 2013), and in line with models of social communication (e.g., Gilbert, Tafarodi, \& Malone, 1993), it is of less relevance here.

Regarding the question whether advice and fluency influence judgments simultaneously or in an either-or fashion, Experiment 1 is less clear. The experimental setup makes it unlikely that participants ignored the advice; yet, for any given decision, participants may allow either the advice or the processing experience to inform their truth judgment. For example, when repeated statements are advised as "false," participants may sometimes follow the advice and sometimes follow fluency, leading to the observed pattern. It is not possible to tease apart these possibilities due to the dichotomous nature of the dependent variable. Experiment 2 solves this problem by using continuous rating scales as a dependent variable and by testing the distribution of the responses using the bimodality coefficient (see below).

\section{Experiment 2}

Experiment 2 replicated Experiment 1's with continuous truth ratings instead of PTJs.

\section{Method}

Participants, Design, and Procedure. We collected data from 41 participants (average age: 24.17 years; 28 female, 13 male). Thus, the design has 4920 observations; similar to Experiment 1 , this yields power to detect effects of Cohen's $d>.315$, for all relevant main effects and interactions, although the factual power is substantially higher (see Experiment 1).

The computer program elicited continuous rather than binary judgments by presenting a scroll bar underneath each statement in the judgment phase. The endpoints were labelled "surely true" and "surely false." Participants could move the pointer on the scroll bar with the mouse and 
proceed self-paced with a "continue" button; upon clicking this button, the program translated the pointer position into values ranging from -50 to +50 , with higher numbers indicating higher judged truth. Everything else was similar to Experiment 1.

\section{Results}

Truth ratings. Figure 2 shows the mean truth ratings. First, we again found a standard truth effect: participants' rated repeated statements' truth higher $(M=5.85, S D=16.09)$ compared to new statements $(M=0.20, S D=14.26), F(1,40)=13.77, p<.001, \eta^{2}{ }_{p}=.256,95 \% C l[.080, .416]$. Second, participants followed the given advice: participants rated statements' truth higher that advisors stated to be "true" $(M=10.46, S D=12.57)$ rather than "false" $(M=-4.41, S D=14.47), F(1,40)=69.15$, $p<.001, \eta_{p}^{2}=.634,95 \% \mathrm{Cl}[.464, .725]$. Again, this advice main effect was qualified by an interaction with advice validity: higher (lower) advice validity led to higher (lower) truth ratings, depending on the "true" /"false" status of the advice, $F(2,80)=18.66, p<.001, \eta^{2}=.318,95 \% \mathrm{Cl}[.173, .426]$. Different from Experiment 1, this interaction effect was due both to judged truth increases (decreases) for "true" advice ("false" advice) from the $50 \%$ to the $60 \%$ level, $F(1,40)=7.50, p=.009, \eta_{p}^{2}=.158,95 \% \mathrm{Cl}$ $[.023, .320]$, and from the $60 \%$ to the $70 \%$ level, $F(1,40)=14.93, p<.001, \eta^{2}=.272,95 \% \mathrm{Cl}[.091, .430]$.

We also observed two non-predicted effects. First, advice had less influence if statements were repeated ("true": $M=12.31, S D=13.03$ vs. "false": $M=-0.61, S D=16.31$ ) compared to new statements ("true": $M=8.60, S D=11.86$ vs. "false": $M=-8.21, S D=11.20), F(1,40)=4.29, p=.045$, $\eta_{\mathrm{p}}^{2}=.097,95 \% \mathrm{Cl}[.001, .251]$. Second, participants provided overall higher truth ratings at higher validity, $F(2,80)=6.04, p=.004, \eta_{\mathrm{p}}^{2}=.131,95 \% \mathrm{Cl}[.028, .235]$, independent of the direction of the advice ("true" vs. "false").

We again analyzed truth ratings for new and repeated statements separately at $50 \%$ and $70 \%$ advice validity; at 50\% validity, both advice, $F(1,40)=20.27, p<.001, \eta^{2}{ }_{p}=.336,95 \% \mathrm{Cl}[.142, .487]$, and repetition, $F(1,40)=8.53, p=.006, \eta_{\mathrm{p}}^{2}=.176,95 \% \mathrm{Cl}[.032, .338]$, influenced participants' truth ratings. At $70 \%$ validity, the repetition influence was reduced but still present, $F(1,40)=6.28, p=.016$, $\eta_{p}^{2}=.136,95 \% \mathrm{Cl}[.014, .296]$, while advice had a substantial impact, $F(1,40)=69.99, p<.001, \eta^{2}{ }_{p}=.636$, $95 \% \mathrm{Cl}[.468, .727]$. Similar to Experiment 1, the difference between new and repeated statements 
was numerically lower in the $70 \%$ validity cases compared to the $50 \%$ validity cases $\left(M_{\text {diff }}=3.25\right.$, $S D=21.45)$; yet, this reduction was again not different from zero, $t(40)=0.95, p=.347$.

Latencies. The same data conditioning and analyses as in Experiment 1 showed similar effects. Participants responded faster to repeated $(M=5737, S D=1000)$ compared to new statements $(M=5969, S D=1040), F(1,40)=18.27, p<.001, \eta_{p}^{2}=.313,95 \% C l[.123, .468]$. As in Experiment 1, participants also responded numerically faster given a "true" advice $(M=5811, S D=933)$ rather than a "false" advice ( $M=5895, S D=977)$, although this effect was not significant in this analysis, $F(1$, $40)=3.91, p=.055, . \eta^{2}{ }^{2}=.089,95 \% \mathrm{Cl}[.000, .241]$.

Either/or vs. joint influence. Experiment 2 allows testing whether participants used advice and fluency simultaneously, or sometimes fluency and sometimes advice. The present design has two consistent cases (i.e., repeated statements advised to be "true"; new statements advised to be "false"), and two inconsistent cases (i.e., repeated statements advised to be "false"; new statements advised to be "true"). The rating distributions of these two cases should substantially differ if the means in Figure 2 sometimes follow fluency and sometimes advice. Consistent case judgments should be unimodal, as both the experiential and declarative information indicate the same response. Inconsistent case judgments should be bimodal; as sometimes fluency should determine the judgments, and sometimes advice, leading to responses at opposite ends of the rating scale. To asses this difference, we estimated the bimodality coefficient $\mathrm{BC}$, which provides a numerical estimate of bimodality (see Freeman \& Dale, 2013). The BC index is computed as (see Pfister et al., 2013):

$$
B C=\frac{\left(\text { skew }^{2}+1\right)}{\text { kurtosis }+3 *\left(\frac{(n-1)^{2}}{(n-2) *(n-3)}\right)}
$$

A distribution's skew indicates the relative size of the distribution's tails, and kurtosis indicates the probability weights in the tails, which correlates with the peakedness of the distribution. In other words, if the relative size of the tails is large relative to the probability of these tails, the distributions is likely to be bimodal. The benchmark for a BC indicating bimodality is .555, 
which would indicate a flat distribution. Values higher than .555 indicate a more bimodal distribution, while values lower than .555 indicate a more unimodal distribution. We separately estimated the BCs for Experiment 2's data distribution of 2460 (41 participants times 60 judgments) truth ratings for consistent cases (i.e., repeated-true and new-false), and of the 2460 statements for the inconsistent statements (i.e., repeated-false and new-true).

Both indices suggest unimodality, $\mathrm{BC}_{\text {consistent }}=.467$ and $\mathrm{BC}_{\text {inconsistent }}=.471$. The critical prediction from an either-or-perspective is, though, that consistent cases should yield a more unimodal distribution, while inconsistent cases should yield a bimodal distribution. For a statistical test, we estimated both indices for each participant, based on 60 judgments each. This analysis yielded similar values for consistent $(M=.497, S D=.145)$ and inconsistent cases $(M=.507, S D=.142)$; these values did not differ from each other, $t(40)=-0.97, p=.336$. Thus, the judgment distributions suggest that participants use both cues simultaneously rather than in an either-or fashion. ${ }^{2}$

\section{Discussion}

Experiment 2 replicated Experiment 1 using continuous ratings. We found again clear influences of processing fluency and advice on truth judgments. Fluency influences were clearly visible even when contradicting advice was available, and even when this advice was supposedly highly valid. We again found no evidence that fluency's influence is significantly reduced when other, potentially contradicting information, is highly valid or vice versa (i.e., the 70\% "false" advice given repeated statements and the $70 \%$ "true" cases given new statements).

Experiment 2 also allowed testing whether fluency and advice have joint influences or an either/or influence by analyzing the response distributions within and across participants. As the bimodality coefficients for consistent and inconsistent judgment cases suggest, Experiment 2's dependent variable does not seem to stem from two different processes.

Again, the fluency influence was not significantly reduced between advice validity levels of $50 \%$ and $70 \%$. This may be attributed to the fact that validity levels of $50 \%$ to $70 \%$ are still quite unreliable so that ample room for repetition and the resulting fluency experience to influence rated 
truth remains. To address this possibility, Experiment 3 replicates Experiment 2 with increased stated validity levels from $70 \%$ to $90 \%$.

\section{Experiment 3}

Experiment 3 replicated Experiment 2 with more extreme advice validities. That is, instead of being correct $50 \%, 60 \%$, and $70 \%$, we increased the communicated correctness to $70 \%, 80 \%$, and $90 \%$.

\section{Method}

Participants, Design, and Procedure. We collected data from 42 participants (average age: 22.33 years; 27 female, 15 male), yielding virtually identical power levels as in Experiment 2 (i.e., 5020 observation with power to detect effects of Cohen's $d>.314$, for all relevant main effects and interactions). Design and procedures were highly similar to Experiment 2.

\section{Results}

Truth ratings. Figure 3 shows the mean truth ratings; as can be seen, Experiment 3 fully replicated Experiment 2. First, we again found a standard truth effect: participants' rated repeated statements' truth higher $(M=7.69, S D=21.30)$ compared to new statements $(M=1.10, S D=20.62), F(1$, 41) $=20.19, p<.001, \eta_{p}^{2}=.330,95 \% \mathrm{Cl}[.139, .480]$. Second, participants followed the given advice: participants rated statements' truth higher that advisors stated to be "true" ( $M=19.15, S D=14.81)$ rather than "false" $(M=-10.36, S D=15.64), F(1,41)=92.25, p<.001, \eta^{2}=.692,95 \% \mathrm{Cl}[.545, .769]$. Again, this advice main effect was qualified by an interaction with advice validity: higher (lower) advice validity led to higher (lower) truth ratings, depending on the "true"/"false" status of the advice, $F(2$, 82) $=19.53, p<.001, \eta_{\mathrm{p}}^{2}=.323,95 \% \mathrm{Cl}[.179, .429]$. As in Experiment 2, this interaction effect was due both to judged truth increases (decreases) for "true" advice ("false" advice) from the $70 \%$ to the $80 \%$ level, $F(1,41)=6.10, p=.018, \eta_{p}^{2}=.130,95 \% \mathrm{Cl}[.013, .287]$, and from the $80 \%$ to the $90 \%$ level, $F(1$, $41)=19.58, p<.001, \eta_{p}^{2}=.323,95 \% \mathrm{Cl}[.133, .474]$.

We also replicated the effect that advice had less influence if statements were repeated ("true": $M=21.46, S D=15.13$ vs. "false": $M=-6.07, S D=17.32$ ) compared to new ("true": $M=16.84$, $S D=14.16$ vs. "false": $M=-14.64, S D=12.41), F(1,41)=5.59, p=.023, \eta_{p}^{2}=.120,95 \% C l[.009, .276]$. 
We again analyzed truth ratings for new and repeated statements separately at $70 \%$ and $90 \%$ advice validity; at $70 \%$ validity, both advice, $F(1,41)=62.43, p<.001, \eta^{2}{ }_{p}=.604,95 \% \mathrm{Cl}[.429, .701]$, and repetition, $F(1,41)=18.87, p<.001, \eta_{p}^{2}=.314,95 \% \mathrm{Cl}[.126, .467]$, influenced participants' truth ratings. At $90 \%$ validity, the repetition influence was still present, $F(1,41)=10.31, p=.003, \eta^{2}=.201,95 \% \mathrm{Cl}$ $[.046, .362]$, while advice had substantial impact, $F(1,41)=92.62, p<.001, \eta_{p}^{2}=.691,95 \% \mathrm{Cl}[.543, .768]$. In addition, at $90 \%$ advice validity, advice had again less influence if statements were repeated ("true": $M=23.74, S D=15.63$ vs. "false": $M=-9.20, S D=18.48$ ) compared to new statements ("true": $M=22.49, S D=14.72$ vs. "false": $M=-18.37, S D=13.37), F(1,41)=7.89, p=.007, \eta_{p}^{2}=.163,95 \% C l[.027$, .323]. Similar to Experiments 1 and 2, the difference between new and repeated statements was numerically lower in the $90 \%$ validity cases compared to the $70 \%$ validity cases $\left(M_{\text {diff }}=5.35\right.$, $S D=18.18$ ); yet, this reduction was again not different from zero on a standard alpha level, $t(41)=1.91, p=.064$

Latencies. The same data conditioning and analyses as in Experiment 2 showed similar effects. Participants responded faster to repeated $(M=5334, S D=1272)$ compared to new statements $(M=5470, S D=1280), F(1,41)=5.90, p=.020, \eta^{2}{ }_{p}=.126,95 \% \mathrm{Cl}[.011, .283]$. As in Experiment 1 , participants also responded faster given a "true" advice $(M=5317, S D=1283)$ rather than a "false" advice $(M=5486, S D=11267), F(1,41)=16.19, p<.001, . \eta_{p}^{2}=.283,95 \% C I[.101, .439]$. In addition, participants responded faster given higher advice validities, $F(2,82)=5.37, p=.007, . \eta_{\mathrm{p}}^{2}=.116,95 \% \mathrm{Cl}$ $[.020, .216]$

Either/or vs. joint influence. As in Experiment 2, the combination of repetition (repeated/new) and validity (true/false) allows categorizing statements as either consistent cases or inconsistent cases. Again, if the means in Figure 4 are due to two separate processes, the distribution of the ratings should substantially differ between the consistent and inconsistent cases. We thus again calculated the bimodality coefficients for consistent and inconsistent cases. Different from Experiment 2, the bimodality coefficients indicated a rather flat distribution, $\mathrm{BC}_{\text {consistent }}=.572$ and $\mathrm{BC}_{\text {inconsistent }}=.557$. However, again, the coefficients apparently not differ between consistent and inconsistent cases. For a statistical test, we again estimated both indices for each participant, based 
on 60 judgments each. This analysis yielded similar values for consistent $(M=.565, S D=.187)$ and inconsistent cases $(M=.550, S D=.184)$; these values did not significantly differ from each other, $t(41)=1.75, p=.087$. If anything, the effect tends to be in the opposite direction; that is, consistent cases are more bimodal than inconsistent cases.

\section{Discussion}

Experiment 3 replicated Experiment 2 with higher advice validities This increase in stated validity did not reduce the repetition effects in Experiment $3\left(\eta^{2}{ }_{p}=.330\right)$ compared to Experiment 2 $\left(\eta_{\mathrm{p}}^{2}=.256\right)$. If anything, the repetition influence was larger and it was still clearly present at $90 \%$ stated validity. Within Experiment 3, the numerical strength of the repetition effect decreased as a function of advice validity; repetition had less impact when advice was highly valid. However, given the present power levels, this decrease was again not significant. Further, the distributions of consistent and inconsistent cases again showed no differential pattern. Across all cases, participants seem to employ both cues (i.e., fluency and advice) in a compensatory fashion, instead of an either/or approach (i.e., sometimes fluency, sometimes advice).

\section{Experiment 4}

The rather surprising finding of Experiments 2 and 3 was that the repetition effect did not significantly diminish at advice levels of high validity; that is, we found no evidence for a validity level by repetition interaction. Experiment 4 therefore replicated Experiment 2 and 3 with a maximal contrast of advice validities. Instead of three advisors with validity levels of $70 \%, 80 \%$, and $90 \%$, participants received information from two advisors; one was guessing (50\% validity) and one was supposedly correct all the time (100\%).

\section{Method}

Participants, Design, and Procedure. We collected data from 38, although one participant was excluded due to a computer failure, leaving 37 participants (average age: 22.84 years; 29 female, 8 male). Design and procedures were highly similar to Experiment 2 with two exceptions. First, in the test phase, participants received information from only two advisors with validity levels of $50 \%$ and $100 \%$. Second, to keep the number of statements per within-condition constant (i.e., old vs. new by 
"true" vs. "false" by validity), participants responded only to 80 statements (i.e., 40 repeated from presentation phase and 40 new statements) in the test phase instead of 120, as Experiment 4 realized only two instead of three validity levels. Thus, the design has 2960 observations in total; this yields power to detect effects of Cohen's $d>.328$, for all relevant main effects and interactions, although the factual power is substantially higher (see Experiment 1). Everything else was identical to previous experiments.

\section{Results}

Truth ratings. Figure 4 shows the mean truth ratings; the pattern is again highly similar to Experiments 2 and 3. First, we again found a standard truth effect. Participants' rated repeated statements' truth higher $(M=5.90, S D=23.62)$ compared to new statements $(M=0.82, S D=22.75), F(1$, $36)=10.74, p=.002, \eta^{2}=.230,95 \% \mathrm{Cl}[.055, .400]$. Second, participants followed the given advice. Participants rated statements' truth higher that advisors stated to be "true" ( $M=17.15, S D=18.79)$ rather than "false" $(M=-10.44, S D=18.76), F(1,36)=56.17, p<.001, \eta_{p}^{2}=.609,95 \% C l[.422, .710]$. This advice main effect was qualified by the expected interaction with advice validity: $50 \%$ vs. $100 \%$ advice validity led to higher (lower) truth ratings, respectively, depending on the "true"/"false" status of the advice, $F(1,36)=39.77, p<.001, \eta_{p}^{2}=.525,95 \% \mathrm{Cl}[.320, .646]$.

The only other effect approaching significance was a theoretically non-relevant three-way interaction (see pattern within Figure 4) of repetition, validity, and "true"/"false" advice, $F(1,36)=$ $4.04, p=.052, \eta_{\mathrm{p}}^{2}=.100,95 \% \mathrm{Cl}[.000, .264]$.

We again analyzed truth ratings for new and repeated statements separately at $50 \%$ and $100 \%$ advice validity. At 50\% validity, both advice, $F(1,36)=18.84, p<.001, \eta^{2}{ }_{p}=.344,95 \% \mathrm{Cl}[.138$, $.500]$, and repetition, $F(1,36)=5.97, p=.020, \eta_{\mathrm{p}}^{2}=.142,95 \% \mathrm{Cl}[.013, .311]$, influenced participants' truth ratings. At $100 \%$ validity, the repetition influence was still present, $F(1,36)=10.99, p=.002$, $\eta_{p}^{2}=.234,95 \% \mathrm{Cl}[.058, .402]$, while advice had clearly the higher impact, $F(1,36)=57.56, p<.001$, $\eta_{p}^{2}=.615,95 \% \mathrm{Cl}[.429, .714]$. Thus, despite the strong influence of advice, the influence of repetition remained reliable across levels of advice validity (i.e., 50\% vs. 100\%), and was numerically (although not statistically) even larger at $100 \%$ advice validity $\left(M_{\text {diff }}=-3.11, S D=20.48\right)$; similar to the previous 
experiments, the difference of the repetition effect between validity levels was not significant, $t(36)=-0.92, p=.361$.

Latencies. The same data conditioning and analyses as in Experiment 2 showed similar effects. Participants responded faster to repeated $(M=6223, S D=907)$ compared to new statements $(M=6400, S D=888), F(1,36)=13.52, p=.001, \eta^{2}{ }_{p}=.273,95 \% \mathrm{Cl}[.084, .438]$. As in previous experiments, participants also responded faster given a "true" advice $(M=6262, S D=878)$ rather than a "false" advice $(M=6361, S D=922), F(1,36)=4.75, p=.036, . \eta_{p}^{2}=.117,95 \% C l[.004, .282]$.

Either/or vs. joint influence. As in Experiments 2 and 3, the combination of repetition (repeated/new) and validity (true/false) allows categorizing statements as either consistent cases or inconsistent cases. Again, if the means in Figure 4 are due to two separate processes, the distribution of the ratings should differ between the consistent and inconsistent cases. Different from previous experiments, the bimodality coefficients indicated bimodal distributions, $\mathrm{BC}_{\text {consistent }}=.571$ and $\mathrm{BC}_{\text {inconsistent }}=.570$ potentially due to more extreme judgments. However, the indices do apparently not differ between consistent and inconsistent cases. For a statistical test, we again estimated both indexes for each participant, based on 40 judgments each. This analysis yielded similar values for consistent $(M=.594, S D=.137)$ and inconsistent cases $(M=.578, S D=.142)$; again, these values did not significantly differ from each other, $t(36)=1.23, p=.228$. Thus, participants seem to jointly use experiential and declarative information in all cases.

\section{Discussion}

Experiment 4 replicated the pattern from Experiments 1 to 3 with a maximal contrast of irrelevant advice (i.e., 50\%) and supposedly deterministic advice (i.e., 100\%). At both levels of validity, the repetition-induced truth effect was clearly there; if anything, the repetition-effect was stronger at $100 \%$ validity $\left(\eta_{\mathrm{p}}^{2}=.234\right)$ compared to $50 \%$ validity $\left(\eta_{\mathrm{p}}^{2}=.124\right)$. And similar to Experiments 2 and 3, the bimodality indices for consistent cases of advice and repetition (i.e., "true"/"false" advice for old/new statements, respectively) and inconsistent cases (i.e., "false"/"true" advice for old/new statements, respectively) suggest that participants used both cues to inform their judgments. 
The obvious question is why participants still use repetition if they receive a supposedly $100 \%$ valid cue. Intuitively, one would expect that the repetition effect is reduced in this condition compared to the $50 \%$ advice validity conditions. The straightforward answer is that participants did not behave fully rational in a Cartesian sense as delineated in the introduction. At $50 \%$, they do not fully ignore the advice, and at $100 \%$, they do not fully heed the advice. That is, even at $100 \%$, there is no ceiling effect and there is still room for an orthogonal cue (i.e., repetition and the resulting fluency experience) to influence the rating. To illustrate this point, we next provide a meta-analytic summary of advice effects and repetition effects.

\section{Meta-Analytic Summary of Experiments 2 to 4}

Experiments 2 to 4 present a unique opportunity to summarize the joint influence of repetition and advice on judged truth. All three experiments use the exact same rating scale and implement advice validity levels from $50 \%$ to $100 \%$ with repeated and new statements. We excluded Experiment 1 as it used binary choices. The only difference is the number of data points on which each estimate is based (e.g., the advice level 70\% appears in Experiment 2 and 3, while the level $80 \%$ appears only in Experiment 3, and so forth). To compute the repetition effect, we calculate the difference of repeated and new statements' truth rating averaged across "true" and "false" advice; for example, the repetition effect at validity level $50 \%$ is then the mean of the difference between repeated and new statements at 50\% "true" advice and 50\% "false" advice. To compute the advice effect, we calculated the difference between "true" and "false" advice averaged across repeated and new statements; for example, the advice effect at validity level $50 \%$ is then the mean of the difference between 50\% "true" advice and 50\% "false" advice for repeated and new statements. Figure 5 plots the respective means for advice and repetition effects.

Figure 5 illustrates several important points. First and foremost, it shows that the repetition effect is highly constant and almost identical across all levels of advice validity, which is consistent with the observation that the repetition effect never interacted with advice validity in any experiment. ${ }^{3}$ Second, participants are highly sensitive to the advice and translate the stated validity directly into their rated truth. Please note that these are raw ratings across 3 experiments; 
nevertheless, the means are almost perfectly linear. Third, participants' usage of the advice cue shows regression to the mean (s. Fiedler \& Unkelbach, 2014). The advice effect at $50 \%$ validity is not zero, and it is no 100 at $100 \%$ validity.

These three points align with the model proposed by Unkelbach and Greifeneder (2013) to explain the data pattern in all experiments. As the influence of the declarative advice cue is regressive to the mean (i.e., neither zero nor one hundred at $50 \%$ and $100 \%$ validity levels), there is room for the experiential fluency cue to influence judgments. Consistent with the notion that feelings and experiential information in general have high subjective truth value (Bless \& Forgas, 2000) participants relied on fluency as a cue across all three experiments. As we varied repetition and thereby fluency only in a 0 to 1 fashion, the repetition effect is almost perfectly constant. The advice influence varies according to the six validity levels across experiments. The resulting pattern in Figure 5 is therefore exactly what one expects from a lens model (Brunswik, 1955; Kareleia and Hogarth, 2008) if two orthogonal cues are combined in a linear fashion.

\section{General Discussion}

The present research investigated the impact of experiential fluency information on truth judgments when declarative information is available. To the best of our knowledge, this is the first investigation to pit the experiential information against a systematically varied declarative source. Previous studies relied either on other internal cues such as existing knowledge (Fazio et al., 2015), source memory (Begg, Anas, and Farrinacci, 1992), or existing percepts (Reber et al., 2004). Here, we relied on declarative information that was directly available at the time of judgment. Based on a cue integration model for fluency effects (Unkelbach \& Greifeneder, 2013), we predicted joint influences of processing fluency and declarative advice information. Four experiments showed this joint and orthogonal influence on judgments of truth. These results critically extend research on processing fluency. In most everyday decisions and judgments, people have additional sources of information available at the time of judgment. If processing fluency only impacted judgments if no additional 
information is available (here: fully ambiguous statements), the importance of processing fluency as a theoretical construct would be substantially diminished.

This extension aligns with the work by Fazio and colleagues (2015), who found that fluency impacts truth ratings even when participants have relevant knowledge. Yet, they still assumed fluency influences judgments only when relevant knowledge was not retrieved. Here, the additional information was constantly available and processing fluency nevertheless influenced judgments.

Another interesting question was whether people use the declarative advice and the experiential fluency information simultaneously (i.e., joint influence) or in an either-or fashion (i.e., fluency sometimes trumps advice). The distributional data suggest that the judgments follow a joint cue integration model, rather than two different strategies (sometimes fluency, sometimes advice). However, this conclusion is based on a null result, as it is logically impossible to show that an outcome is not based on two processes instead of a single process.

Finally, the data suggest that people are not "rational" in a Cartesian sense, but rather always use their internally available cues to inform their judgments. That is, even when a statement is clearly labelled as false, people still believe it somewhat more if it is fluently processed. This point was recently highlighted by Pennycook, Cannon, and Rand (in press), who showed that previous exposure increases believability of rather unlikely fake news; although the increases were rather small, they were statistically highly reliable. The present data even goes one step further and suggest that this non-rationality goes both ways. At 50\% advice validity (i.e., guessing), people do not fully ignore the advice, and at $100 \%$, they do not fully ignore the experience. That is, similar to the impossibility to ignore experiential information, it appears that people cannot help but use declarative information clearly labelled as non-informative.

There are also four caveats. First, we relied on a single manipulation of the experiential cue; that is, repetition. While this is the typical variation in a truth judgment paradigm, it might be interesting to see if other manipulations of processing fluency show the same consistency, especially as not all fluency manipulation appear to be similar in their effects (see Silva, Garcia-Marques, \& Reber, 2017). Second, all experiments created situations in which only two cues are relevant: fluency 
and advice, assuming that participants had little to no knowledge. This situation favors linear cue integration. As Payne, Bettman, \& Johnson (1993) noted, linear models do not adequately describe human judgment when the number of relevant cues is larger than three, and it is a distinct possibility that in such cases, people may use a more heuristic style to reduce the complexity of the judgment situation (e.g., always and only use advice).

Third, Unkelbach and Rom (2017) recently argued that referential networks rather than processing fluency may underlie repetition-induced truth effects. However, their theory still built on Brunswik's lens model and still allowed for fluency to influence judgments of truth. Thus, the present argument is not in contradiction with their theory.

And fourth, we found no evidence for cue interactions. All experiments showed no significant interaction between advice validity and repetition-induced fluency (see also Footnote 3). Intuitively, one would expect that increases in advice validity reduces the processing experience's impact. This intuition is backed-up by the thought experiment that one would not expect repetition to influence the truth judgment that "Osorno is a city in Chile" if someone has, for example, visited Osorno in Chile. Our meta-analytic summary partially provides an answer. Participants might have discounted the advice, which is rather unlikely given that advice even had an effect at $50 \%$. Rather, the advice influence shows regression to the mean (Galton, 1886), which, in particular at high advice levels, leaves enough room on the rating scale for the orthogonal experiential cue to influence judgments.

The intriguing implication of the present data is that the fluency influence might be constantly there at any level of declarative information (see Figure 5), which might explain many apparently irrational behaviors or beliefs as a reliance on internal cues rather than externally provided information (e.g., beliefs that vaccinations cause autism); that is, fluency might even make a clearly false statement more believable.

\section{Conclusion}

In line with a lens model of cue integration, the present research shows that experiential fluency impacts judgments and decisions even when declarative information about the to-be-judged stimulus is available. It thereby substantiates the role of processing fluency as an important factor in 
Fluency_and_Advice 23

judgments and decisions, and it suggests that knowing and feeling might not be so different after all when it comes to judging truth. 


\section{References}

Begg, I. M., Anas, A., \& Farinacci, S. (1992). Dissociation of processes in belief: Source recollection, statement familiarity, and the illusion of truth. Journal of Experimental Psychology: General, $121,446-458$.

Bless, H., \& Forgas, J. P. (2000). The message within: Toward a social psychology of subjective experiences. In H. Bless \& J. P. Forgas (Eds.), The message within: The role of subjective experience in social cognition and behavior (pp. 372-392). Philadelphia, PA: Psychology Press.

Brunswik, E. (1952). The conceptual framework of psychology. Chicago: University of Chicago Press.

Brunswik, E. (1955). Representative design and probabilistic theory in a functional psychology. Psychological Review, 62, 193-217.

Dechêne, A., Stahl, C., Hansen, J., \& Wänke, M. (2010). The truth about the truth: A meta-analytic review of the truth effect. Personality and Social Psychology Review, 14, 238-257.

Fazio, L. K., Brashier, N. M., Payne, B. K., \& Marsh, E. J. (2015). Knowledge does not protect against illusory truth. Journal of Experimental Psychology: General, 144, 993-1002.

Feustel, T, Shiffrin, R., \& Salasoo, A. (1983). Episodic and lexical contributions to the repetition effect in word recognition. Journal of Experimental Psychology: General, 112, 309-346.

Fiedler, K., \& Unkelbach, C. (2014). Regressive judgment: Implications of a universal property of the empirical world. Current Directions in Psychological Science, 23, 361-367.

Freeman, J. B., \& Dale, R. (2013). Assessing bimodality to detect the presence of a dual cognitive process. Behavior Research Methods, 45, 83-97.

Galton, F. (1886). Regression towards mediocrity in hereditary stature. Journal of the Anthropological Institute of Great Britain and Ireland, 15, 246-263.

Gilbert, D. T., Tafarodi, R. W., \& Malone, P. S. (1993). You can't not believe everything you read. Journal of Personality and Social Psychology, 65, 221-233.

Greifeneder, R., Alt, A., Bottenberg, K., Seele, T., Zelt, S., \& Wagener, D. (2010). On writing legibly: Processing fluency systematically biases evaluations of handwritten material. Social Psychological and Personality Science, 1, 230-237. 
Greifeneder, R., Bless, H., \& Scholl, S. (2013). About swift defaults and sophisticated safety nets: A process perspective on fluency's validity in judgment. In C. Unkelbach \& R. Greifeneder (Eds.), The experience of thinking: How the fluency of mental processes influences cognition and behavior (pp. 220-233). Hove: Psychology Press.

Greifeneder, R., Bless, H., \& Pham, M. T. (2011). When do people rely on affective and cognitive feelings in judgment? A review. Personality and Social Psychology Review, 15, 107-141.

Hansen, J., Dêchene, A., \& Wänke, M. (2008). Discrepant fluency increases subjective truth. Journal of Experimental Social Psychology, 44, 687-691.

Hertwig, R., Herzog, S. M., Schooler, L. J., \& Reimer, T. (2008). Fluency heuristic: A model of how the mind exploits a by-product of information retrieval. Journal of Experimental Psychology: Learning, Memory, and Cognition, 34, 1191-1206.

Herzog, S. M., \& Hertwig, R. (2013). The ecological validity of fluency. In C. Unkelbach, R. Greifender (Eds.), The experience of thinking: How the fluency of mental processes influences cognition and behaviour (pp. 190-219). New York: Psychology Press.

Hilbig, B. E. (2012). How framing statistical statements affects subjective veracity: Validation and application of a multinomial model for judgments of truth. Cognition, 125, 37-48.

Jacoby, L. L., Allan, L. G., Collins, J. C., \& Larwill, L. K. (1988). Memory influences subjective experience: Noise judgments. Journal of Experimental Psychology: Learning, Memory, and Cognition, 142, 240-247.

Karelaia, N., \& Hogarth, R. (2008). Determinants of linear judgment: A meta-analysis of lens model studies. Psychological Bulletin, 134, 404-426.

Kromrey, J. D., \& Bell, B. A. (2010). ES_ANOVA: A SAS macro for computing point and interval estimates of effect sizes associated with analysis of variance models. SouthEast SAS Users Group.

Olds, J. M., \& Westerman, D. L. (2012). Can fluency be interpreted as novelty? Retraining the interpretation of fluency in recognition memory. Journal of Experimental Psychology: Learning, Memory, and Cognition, 38, 653-664. 
Pennycook, G., Cannon, T. D., \& Rand, D. G. (in press) Prior exposure increases perceived accuracy of fake news. Journal of Experimental Psychology: General.

Pfister, R., Schwarz, K. A., Janczyk, M., Dale, R., \& Freeman, J. (2013). Good things peak in pairs: a note on the bimodality coefficient. Frontiers in Psychology, 4, 700.

Reber, R., \& Schwarz, N. (1999). Effects of perceptual fluency on judgments of truth. Consciousness and Cognition, 8, 338-342.

Reber, R., \& Unkelbach, C. (2010). The epistemic status of processing fluency as source for judgments of truth. Review of Philosophy and Psychology, 1, 563-581.

Reber, R., Winkielman, P., \& Schwarz, N. (1998). Effects of perceptual fluency on affective judgments. Psychological Science, 9, 45-48.

Reber, R., Zimmermann, T. D., \& Wurtz, P. (2004). Judgments of duration, figure-ground contrast, and size for words and nonwords. Perception and Psychophysics, 66, 1105-1114.

Scholl, S., Greifeneder, R., \& Bless, H. (2014). When fluency signals truth: Prior successful reliance on fluency moderates the impact of fluency on truth judgments. Journal of Behavioral Decision Making, 27, 268-280.

Silva, R. R., Garcia-Marques, T., \& Reber, R. (2017). The informative value of type of repetition: Perceptual and conceptual fluency influences on judgments of truth. Consciousness and Cognition, 51, 53-67.

Topolinski, S., \& Deutsch, R. (2013). Phasic affective modulation of semantic priming. Journal of Experimental Psychology: Learning, Memory, and Cognition, 39, 414-436.

Tversky, A., \& Kahneman, D. (1973). Availability: A heuristic for judging frequency and probability. Cognitive Psychology, 5, 207-232.

Unkelbach, C. (2006). The learned interpretation of cognitive fluency. Psychological Science, 17, 339345.

Unkelbach, C. (2007). Reversing the truth effect: Learning the interpretation of processing fluency in judgments of truth. Journal of Experimental Psychology: Learning, Memory, and Cognition, 33, 219-230. 
Unkelbach, C., Fiedler, K., Bayer, M., Stegmüller, M., \& Danner, D. (2008). Why positive information is processed faster: The density hypothesis. Journal of Personality and Social Psychology, 95, 36-49.

Unkelbach, C., \& Stahl, C. (2009). A multinomial modeling approach to dissociate different components of the truth effect. Consciousness and Cognition, 18, 22-38.

Unkelbach, C., \& Topolinski, S. (2017). The overlooked relation of reliability and statistical power. Manuscript submitted for publication.

Unkelbach, C., von Hippel, W., Forgas, J. P., Robinson, M. D., Shakarchi, R. J., \& Hawkins, C. (2010). Good things come easy: Subjective exposure frequency and the faster processing of positive information. Social Cognition, 28, 538-555.

Unkelbach, C., \& Greifeneder, R. (2013). A general model of fluency effects in judgment and decision making. In C. Unkelbach \& R. Greifeneder (Eds.), The experience of thinking: How the fluency of mental processes influences cognition and behaviour (pp. 11-32). New York, NY: Psychology Press.

Unkelbach, C., \& Rom, S. C. (2017). A referential theory of the repetition-induced truth effect. Cognition, 160, 110-126.

Vanhove, J. (2017). Abandoning standardised effect sizes and opening up other roads to power. http://janhove.github.io/design/2017/07/14/OtherRoadsToPower (retrieved Oct. 24th, 2017).

Westfall, J., Kenny, D. A., \& Judd, C. M. (2014). Statistical power and optimal design in experiments in which samples of participants respond to samples of stimuli. Journal of Experimental Psychology: General, 143, 2020-2045.

Westfall, J. (2018). PANGEA (v0.2). Power ANalysis for GEneral Anova designs. https://jakewestfall.shinyapps.io/pangea/ (retrieved June $25^{\text {th }}, 2018$ ). 


\section{Footnotes}

1. The predicted additive nature of fluency influences is undisputed when people judge inherently experiential properties. For example, when participants judged noise levels while reading old or new statements, the increased fluency due to repetition leads to lower judged noise, in addition to a clear main effect of factual noise volume (Jacoby, Allan, Collins, \& Larwill, 1988). Similarly, when judging presentation duration, participants judged fluently processed stimuli (i.e., words) as being presented longer than less fluently processed stimuli (i.e., non-words), again, in addition to a main effect of factual presentation time.

2. Experiment 1 did not allow testing this, as the binary DV forces bimodal distributions. Accordingly, the bimodality coefficients for Experiment 1's DV distributions clearly indicate bimodality, with $\mathrm{BC}_{\text {consistent }}=.856$ and $\mathrm{BC}_{\text {inconsistent }}=.859$.

3. The meta-analytic summary also allows testing the difference of repetition effects at low levels of advice and high levels of advice with $n=120$ from Experiment 2 to 4 . This analysis shows that there is a numerically reduction from high to low advice validity, $\left(M_{\text {diff }}=2.02, S D=20.34\right)$; yet, even given the high power of the pooled experiments, this difference was not significant, $t(119)=1.09, p=.279$. 


\section{Figure 1}

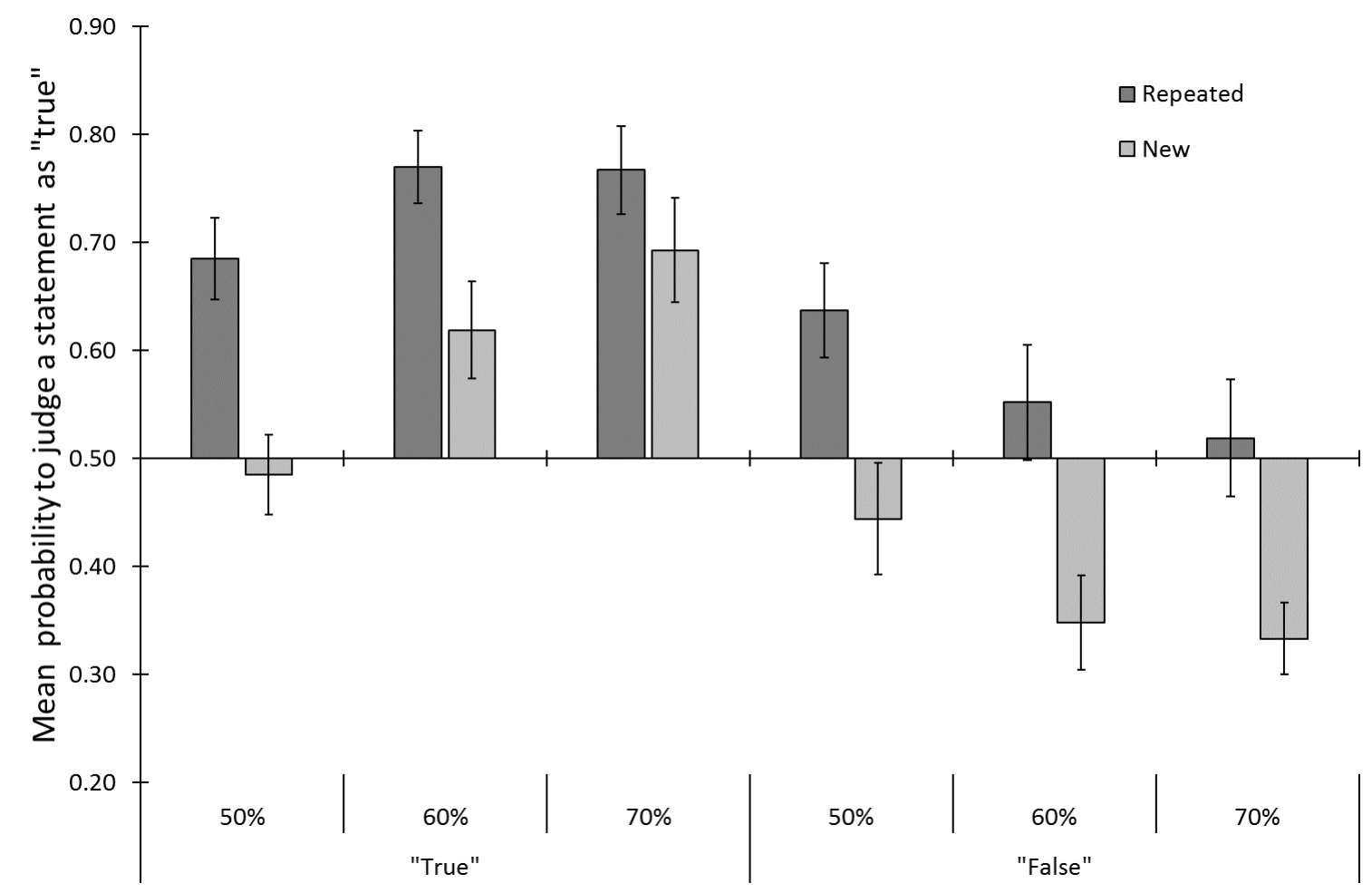

Figure 1. Experiment 1's mean probabilities to judge a statement as true (PTJ) as a function of repetition (repeated vs. new), advice ("true" vs. "false"), and advice validity (50\% vs. $60 \%$ vs. $70 \%$ ). Error bars represent standard errors of the means. 
Figure 2

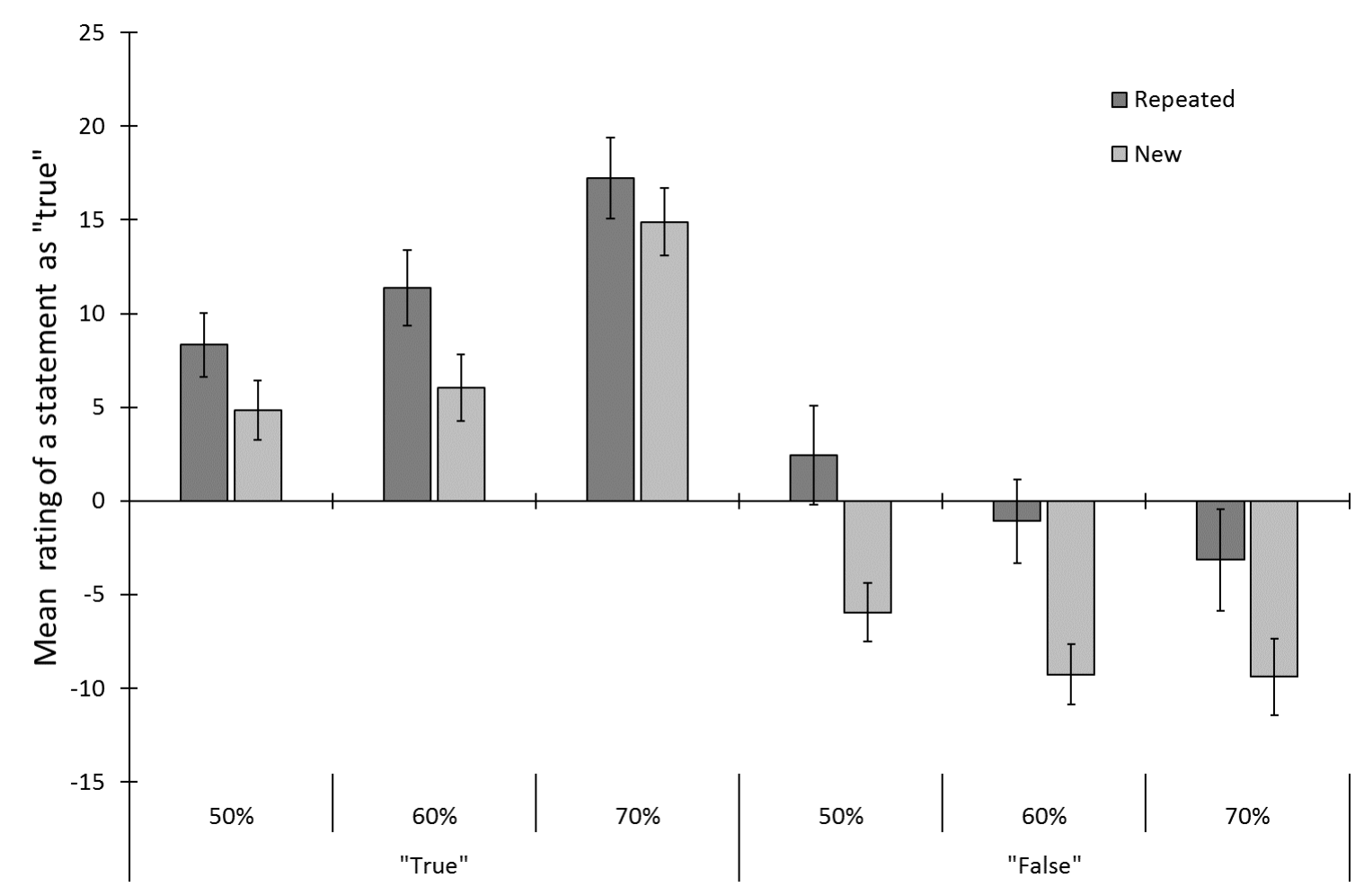

Figure 2. Experiment 2's mean truth ratings as a function of repetition (repeated vs. new), advice ("true" vs. "false"), and advice validity ( $50 \%$ vs. $60 \%$ vs. $70 \%$ ). Error bars represent standard errors of the means. 
Figure 3

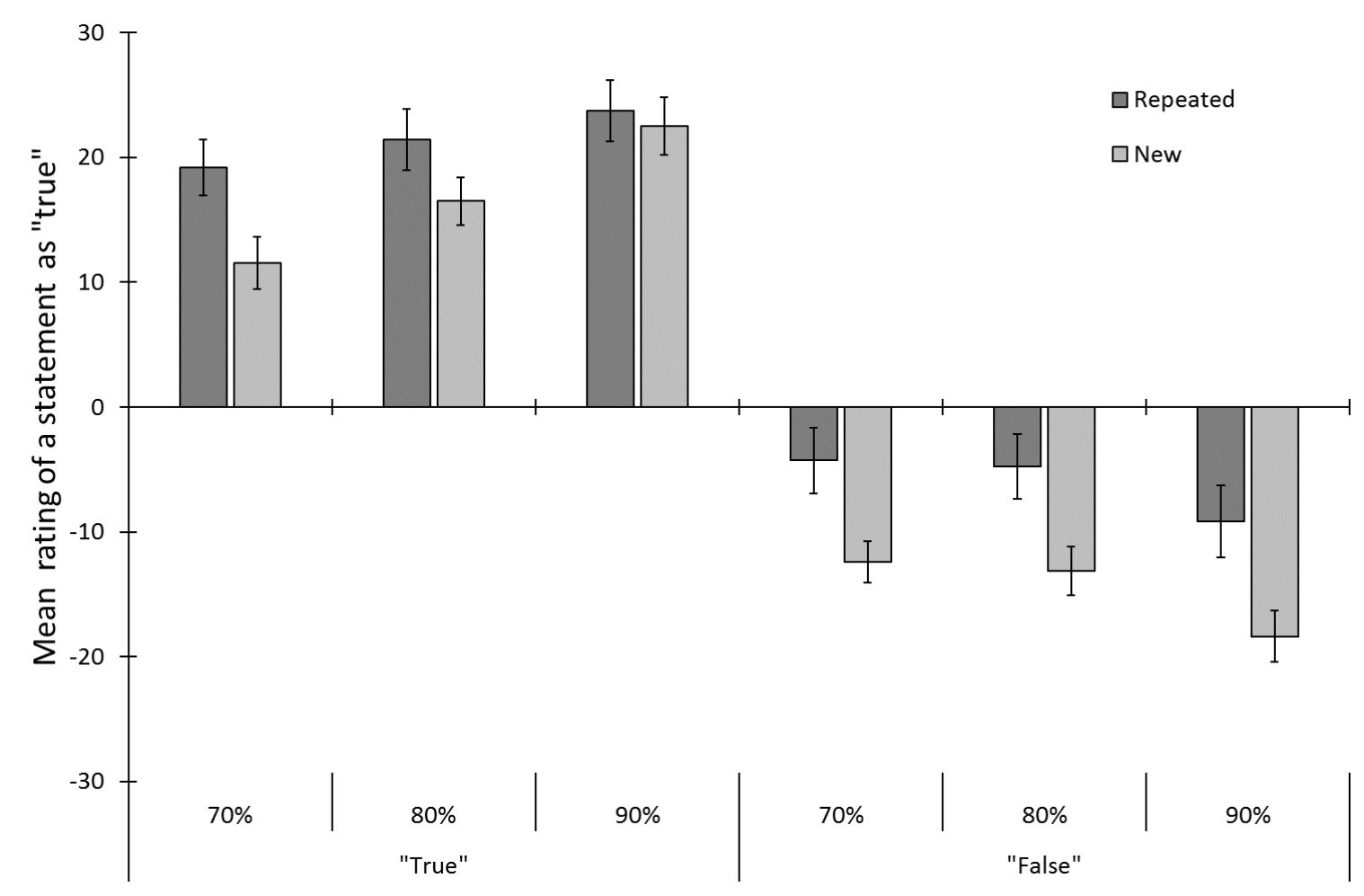

Figure 3. Experiment 3's mean truth ratings as a function of repetition (repeated vs. new), advice ("true" vs. "false"), and advice validity (70\% vs. $80 \%$ vs. $90 \%$ ). Error bars represent standard errors of the means. 
Figure 4

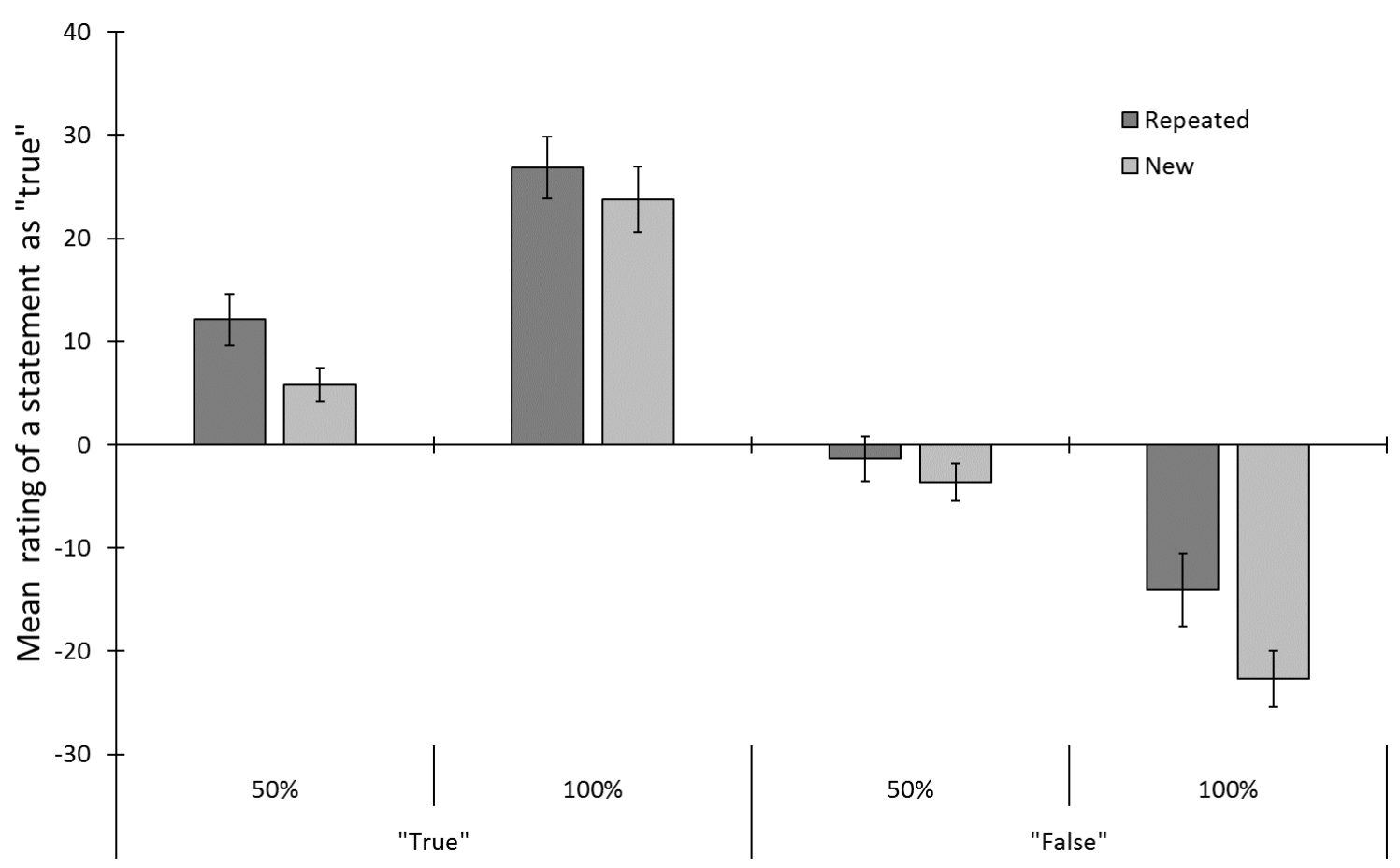

Figure 4. Experiment 4's mean truth ratings as a function of repetition (repeated vs. new), advice ("true" vs. "false"), and advice validity ( $50 \%$ vs. $100 \%$ ). Error bars represent standard errors of the means. 


\section{Figure 5}

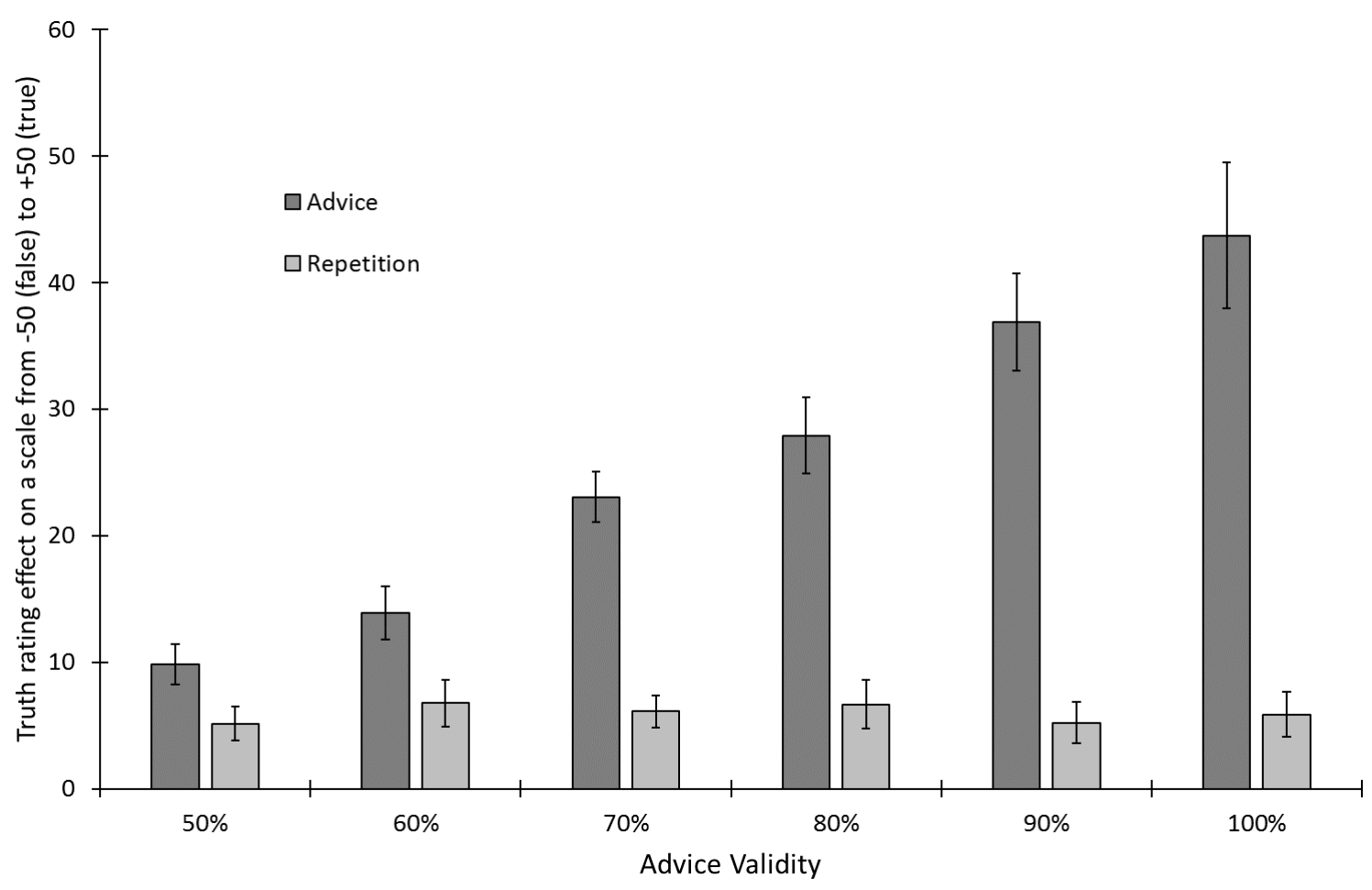

Figure 5. Truth ratings effects of advice (i.e., averaged across repeated and new statements for the difference between "true" and "false" advice) and repetition (i.e., averaged across "true" and "false" advice for the difference between repeated and new statements) as a function of advice validity (50\% to $100 \%)$. The means are based on participants' raw ratings from three experiments with the same rating scale in which the advice validity level appeared $(50 \%-n=78,60 \%-n=41,70 \%-n=83$, $80 \%-n=42,90 \%-n=42$, and $100 \%-n=37)$. Error bars represent standard errors of the means. 